\title{
Odorant-Evoked Nitric Oxide Signals in the Antennal Lobe of Manduca sexta
}

\author{
Chad Collmann, ${ }^{1}$ Mikael A. Carlsson, ${ }^{2}$ Bill S. Hansson, ${ }^{2}$ and Alan Nighorn ${ }^{1}$ \\ ${ }^{1}$ Arizona Research Laboratories, Division of Neurobiology, University of Arizona, Tucson, Arizona 85721, and 2Division of Chemical Ecology, Department \\ of Crop Science, Swedish University of Agricultural Sciences, S-230 53 Alnarp, Sweden
}

The gaseous signaling molecule nitric oxide (NO) can affect the activities of neurons and neural networks in many different systems. The strong expression of NO synthase (NOS) in the primary synaptic neuropil (the antennal lobe in insects and the olfactory bulb in vertebrates) of the olfactory system of most organisms, and the unique spheroidal geometry of olfactory glomeruli in those neuropils, have led to suggestions that NO signaling is important for processing olfactory information. No direct evidence exists, however, that NO signals are produced in olfactory glomeruli. We investigated the production of NO in the antennal lobe of the moth, Manduca sexta, by using immunocytochemistry and real-time optical imaging with a NO-sensitive fluorescent marker, diaminofluorescein diacetate. We confirmed that NOS was expressed in the axons of olfactory receptor neurons projecting to all glomeruli. Soluble guanylyl cyclase, the best characterized target of NO, was found in a subset of postsynaptic antennal lobe neurons that included projection neurons, a small number of GABA-immunoreactive neurons, and a serotonin-immunoreactive neuron. We found that odorant stimulation evoked NO signals that were reproducible and spatially focused. Different odorants evoked spatially distinct patterns of NO production. Increased concentrations of pheromone and plant odorants caused increases in peak signal intensity. Increased concentrations of plant odorants also evoked a dramatic increase in signal area. The results of these experiments show clearly that odorant stimulation can evoke NO production in the olfactory system. The NO signals produced are likely to play an important role in processing olfactory information.

Key words: diaminofluorescein; nitric oxide synthase; moth; olfactory; optical imaging; soluble guanylyl cyclase

\section{Introduction}

The gaseous messenger nitric oxide (NO) is thought to play an important role in processing olfactory information in many different species. This is attributable partly to the structural organization of the first synaptic neuropil, in which primary afferents from the sensory periphery make synapses with local secondary cells in spheroidal bundles of neuropil called glomeruli. Although the reason for this relatively unique structural arrangement is unknown, it has been hypothesized that its form is ideally suited to regulate the activities of gaseous messengers, such as NO, the actions of which are limited by diffusion rather than synaptic connections (Breer and Shepherd, 1993).

Results of localization studies for NO synthase (NOS) strengthen this hypothesis. Using a combination of NADPH diaphorase staining and immuncytochemistry, evidence for NOS expression has been found in the antennal lobes (ALs)/olfactory bulbs of many vertebrate and invertebrate models including ro-

Received Feb. 27, 2004; revised May 5, 2004; accepted May 22, 2004.

This work was supported by National Institutes of Health-National Institute on Deafness and Other Communication Disorders Grant DC04292 to A.N. and European Union: Future and Emerging Technologies Artificial Math Grant IST-2001-33066 to B.S.H. We thank Shahan Fernando, Mark Higgins, and Caroline Wilson for critical review of this manuscript. We appreciate the gift of the anti-GABA antiserum from Dr. Irina Sinakevitch. We also thank Patricia Jansma for assistance with confocal microscopy, Dr. A.A. Osman for rearing M. sexta at the Arizona Research Laboratories Division of Neurobiology, and Dr. Joachim Schachtner for supplying us with additional M. sexta.

Correspondence should be addressed to Dr. Alan Nighorn, The University of Arizona, Room 611 Gould-Simpson Building, Tucson, AZ 85721. E-mail: nighorn@neurobio.arizona.edu.

DOI:10.1523/JNEUROSCI.0710-04.2004

Copyright $\odot 2004$ Society for Neuroscience $\quad$ 0270-6474/04/246070-08\$15.00/0 dents, primates, insects, and mollusks (Bredt et al., 1991; Elphick et al., 1995; Muller and Hildebrandt, 1995; Hopkins et al., 1996; Kendrick et al., 1997; Alonso et al., 1998; Nighorn et al., 1998; Murakami et al., 2004). Many of those studies also found well characterized targets of NO, such as soluble guanylyl cyclase (sGC), colocalized with NOS.

Taken together, these studies show that both NOS and potential targets of NO are expressed highly in the olfactory systems of many different organisms. This consistent and widespread colocalization suggests that NO signaling is likely to be important for olfactory function. Indeed, exogenous application of NO has been shown to increase the frequency of local field potential oscillations in the procerebral lobe of the mollusk, Limax maximus (Gelperin et al., 2000). No study to date, however, has provided direct evidence that NO is produced in the olfactory system in response to odorant stimuli. Here, we used the olfactory system of the moth, Manduca sexta, as a model to investigate odorantevoked NO production. We have shown previously that NOS and sGC mRNA is expressed in the adult and developing olfactory system of M. sexta (Nighorn et al., 1998; Gibson and Nighorn, 2000). In this study, we used immunocytochemistry to label NOS protein in the AL and found that it is localized to olfactory receptor neuron (ORN) axons. We found also that the $\alpha$ subunit of sGC is expressed in a subset of intrinsic AL neurons that includes projection neurons, a unique serotonergic interneuron, and a small number of GABAergic neurons. Taken together, these immunocytochemical labeling patterns suggested strongly that NO 
might be produced when ORNs are activated by odorant stimuli. To investigate this, we used real-time imaging with an NOsensitive dye to show that $\mathrm{NO}$ was produced in the AL when the antenna was stimulated with different odorants. The patterns of NO production were spatially focused and depended strongly on the identity and concentration of the odorant stimulus. This is the first direct demonstration that odorant stimulation causes NO production in the olfactory system.

\section{Materials and Methods}

Animals and reagents. M. sexta (Lepidoptera: Sphingidae) were reared in a laboratory culture on an artificial diet as described previously (Sanes and Hildebrand, 1976). For these experiments, adults, 1-3 d posteclosion, were used. Before dissection, animals were anesthetized on ice. All reagents were purchased from Sigma (St. Louis, MO) unless indicated otherwise.

Western blotting analysis of M. sexta $s G C \alpha$ subunit 1 antiserum. Adult brains were homogenized in NuPage LDS Sample Buffer ( $\mathrm{pH}$ 8.5; Invitrogen, Gaithersburg, MD) containing $0.5 \mathrm{M}$ DTT and a protease inhibitor mixture [4-(2 aminoethyl) benzenesulfonyl fluoride, transepoxysuccinyl-L-leucylamido(4-guanidino)butane, bestatin, leupeptin, aprotinin, and sodium EDTA; $0.1 \mathrm{mg} / \mathrm{ml}$. Samples were separated by SDS-PAGE and transferred to Immobilon-P membrane (Millipore, Billerica, MA). Anti-M. sexta sGC $\alpha$ subunit 1 (MsGC $\alpha 1)$ custom polyclonal antiserum was generated in goats (Bethyl Laboratories Inc., Montgomery, TX) against the peptide CIREALKDYGIGQANSTDVDT and was applied to the blot at a 1:2500 dilution in TBS containing 20\% Tween 20 detergent. The secondary antibody (anti-goat HRP; Jackson ImmunoResearch, West Grove, PA) was used at a 1:10,000 dilution, and the blot was developed with an Opti-4CN kit (Bio-Rad, Hercules, CA).

Immunocytochemistry. Brains were fixed with $4 \%$ paraformaldehyde in $0.1 \mathrm{M}$ sodium PBS overnight at $4^{\circ} \mathrm{C}$, then embedded in agarose and sectioned with a vibrating microtome. Sectioned tissue was blocked with $5 \%$ normal donkey serum and $0.5 \%$ Triton X-100 in PBS for $1 \mathrm{hr}$ at room temperature. For GABA labeling, tissue was reduced with $0.13 \mathrm{~m}$ sodium borohydride before sectioning, then blocked with $10 \%$ normal donkey serum, $0.5 \%$ sodium metabisulfate, and $0.5 \%$ Triton X-100 in $0.05 \mathrm{M}$ Tris-HCl. Primary antisera were diluted in blocking solution and applied overnight at room temperature as follows: goat anti-MsGC $\alpha 1,1: 2500$; rabbit anti-universal NOS monoclonal antisera (Oncogene Research Products, Golden, CO), 1:150; rabbit anti-GABA polyclonal antisera (generously supplied by Dr. Irina Sinakevitch, University of Arizona, Tucson, AZ), 1:100; and rabbit anti-5-HT polyclonal antisera (Incstar, now DiaSorin, Stillwater, MN), 1:4000. The fluorescent secondary antibodies used were donkey anti-rabbit FITC and donkey anti-goat $\mathrm{Cy} 3$ (Jackson ImmunoResearch), each diluted 1:250 in blocking solution and applied overnight at $4^{\circ} \mathrm{C}$. Sections were mounted in $60 \%$ glycerol in PBS. Images were collected with a Nikon PCM 2000 laser-scanning confocal microscope equipped with a Nikon E800 microscope and operated by Simple PCI software (C-Imaging Systems, Cranberry Township, PA). Images were processed and false-colored with Simple PCI software.

Optical recordings. Animals were restrained in plastic tubes and immobilized with dental wax. A window was cut in the head between the eyes. Glands, muscles, and trachea were removed to allow for observations of the ALs. The NO-sensitive dye 4-amino-5-methylamino-2', $7^{\prime}$-difluorofluorescein diacetate (DAF-FM DA; Calbiochem, La Jolla, CA) was dissolved in a detergent, 20\% Pluronic F-127 in DMSO (Molecular Probes, Eugene, OR), then diluted to $10 \mu \mathrm{M}$ in moth saline (in mM: $150 \mathrm{NaCl}, 3 \mathrm{KCl}, 3 \mathrm{CaCl}_{2}, 10$ $\mathrm{N}$-tris(hydroxymethyl)methyl-2-aminoethanesulfonic acid buffer, and 25 sucrose, $\mathrm{pH}$ 6.9). In the final preparation, DMSO was present at $0.4 \%(\mathrm{v} / \mathrm{v})$ and Pluronic F-127 at $0.04 \%(\mathrm{v} / \mathrm{v})$. The dye solution was applied to the ALs for $60 \mathrm{~min}$ at room temperature, then replaced with normal moth saline and rinsed in normal saline for $5 \mathrm{~min}$. Recordings were then done immediately in vivo, and the saline was replaced continuously during the experiments. Pharmacological antagonists of NO signals were used during some experiments to test the specificity of the dye. We used a mixture of NO-signaling antagonists that contained 2-(4-carboxyphenyl)-4,4,5,5-tetramethylimidazoline1-oxyl 3 oxide (C-PTIO; Dojindo, Gaithersburg, MD) diluted to $100 \mu \mathrm{M}$ and
$\mathrm{N}$-nitro-L-arginine methyl ester (L-NAME) diluted to $1 \mathrm{~mm}$ in moth saline and applied for $30 \mathrm{~min}$ before resuming the recordings.

We used an imaging system from TILL Photonics (Gräfelfing, Germany) and an upright microscope (Olympus, Hamburg, Germany) to record optical responses. Filter settings were dichroic: $500 \mathrm{~nm}$; emission long pass, $515 \mathrm{~nm}$; excitation, $488 \mathrm{~nm}$. Sequences of 60 frames ( 4 frames/ sec; 200 msec exposure) were recorded through a $10 \times$ (W 0,30; Olympus) water immersion objective. Odorant stimulation started at frame 12 and consisted of a series of six 100 msec odorant pulses with an interstimulus period of $100 \mathrm{msec}$. Images were binned $2 \times$ on chip (to $320 \times$ 240 pixels) to increase the signal/noise ratio. Protocol execution and data analyses were done with TILLvisION 4.0 software (TILL Photonics).

Odorant delivery. Charcoal-filtered and moistened continuous air stream $(30 \mathrm{ml} / \mathrm{sec})$ ventilated the antenna ipsilateral to the recorded $\mathrm{AL}$ through a glass tube $(7 \mathrm{~mm}$ inner diameter) at a distance of $10 \mathrm{~mm}$ from the antenna. An empty Pasteur pipette was inserted through a small hole in the glass tube, blowing an air stream of $15 \mathrm{ml} / \mathrm{sec}$. Air was blown (15 $\mathrm{ml} / \mathrm{sec}$ ) through an odorant-containing pipette by a computercontrolled puffer device (Syntech, Hilversum, The Netherlands) into the continuous air stream. During stimulation, the air stream $(15 \mathrm{ml} / \mathrm{sec})$ was switched from the empty pipette to the odorant-containing one to minimize mechanical influences caused by adding air. Odorants were diluted in paraffin oil (plant-derived odorants) or cyclohexane (pheromone components), and $10 \mu \mathrm{l}$ of odorant solution was applied on filter papers $(5 \times 15 \mathrm{~mm})$. Filter papers were inserted in Pasteur pipettes sealed with Parafilm (American National Can, Chicago, IL) and stored at $-20^{\circ} \mathrm{C}$ until the start of an experiment. For control stimuli, we used filter papers that contained $10 \mu \mathrm{l}$ of solvent only. The odorants tested were geraniol, 1-octanol, (+/-)-linalool, phenylacetaldehyde (PAA), and the two primary sexual pheromone components E10,Z12-hexadecadienal (bombykal) and E10,E12,Z12-hexadecatrienal (EEZ; a synthetic mimic). The pheromone components were tested primarily in a 1:1 mixture. The purities of the odorants were between 95 and $99 \%$. Linalool was tested in a decadic concentration series from $10 \mathrm{ng}$ to $1 \mathrm{mg}$. The pheromone mixture was tested in a decadic concentration series from $1 \mathrm{ng}$ to $1 \mu \mathrm{g}$. All other plant odorants were tested at $10 \mu \mathrm{g}$. Note, however, that because odorants were delivered in a constant air stream and the relative saturation within odorant cartridges may not increase linearly with odorant concentration, the actual amount of odorant that reached the antenna is likely to be lower and may not have scaled linearly with increasing concentration.

Data analyses. Background fluorescence $(F)$ was defined as an average of frames 2-11 (prestimulation frames). $F$ was subtracted from all frames to yield a change in fluorescence $(\mathrm{d} F)$, and signals were expressed as a relative change $(\mathrm{d} F / F)$. To correct for photo-bleaching, a sequence with pure air stimulation was expressed first as $\mathrm{d} F / F$, then subtracted from a sequence with odorant stimulation. Air-stimulus subtraction also controlled for any possible mechanical component of the signal caused by switching the air stream from one pipette to another. All $\mathrm{d} F / F$ values reported are expressed as percentages.

To calculate $\mathrm{d} F / F$ specifically from active glomeruli, one region of interest $(10 \times 10$ pixels, corresponding to $\sim 20 \times 20 \mu \mathrm{m})$ was analyzed from each glomerulus that showed activity. Several regions of interest were used to analyze the macroglomerular complex (MGC) area because of its size. To calculate $\mathrm{d} F / F$ from controls, the $x-y$ positions of regions of interest from recordings of odorant-evoked signals were copied to recordings of controls. The temporal dynamics and the values of $\mathrm{d} F / F$ for each signal were determined from one region of interest chosen from the glomerulus activated most highly by each stimulus. The mean pixel value for a region of interest was calculated for each frame of a bleachingcorrected sequence. To visualize the temporal dynamics of a signal, we plotted the mean pixel value for each frame against time. To compare the temporal dynamics of the signals for the different odorants tested, an average signal was calculated for each odorant (10 $\mu \mathrm{g}$ of plant-derived odorants, $1 \mu \mathrm{g}$ of pheromone, and $10 \mu \mathrm{l}$ of control solvent), then plotted against time.

The peak of a signal was calculated as an average of the mean pixel values for four frames at the peak of activity (frames 20-23 for plantderived odorants and frames 23-26 for pheromone) of a bleaching- 

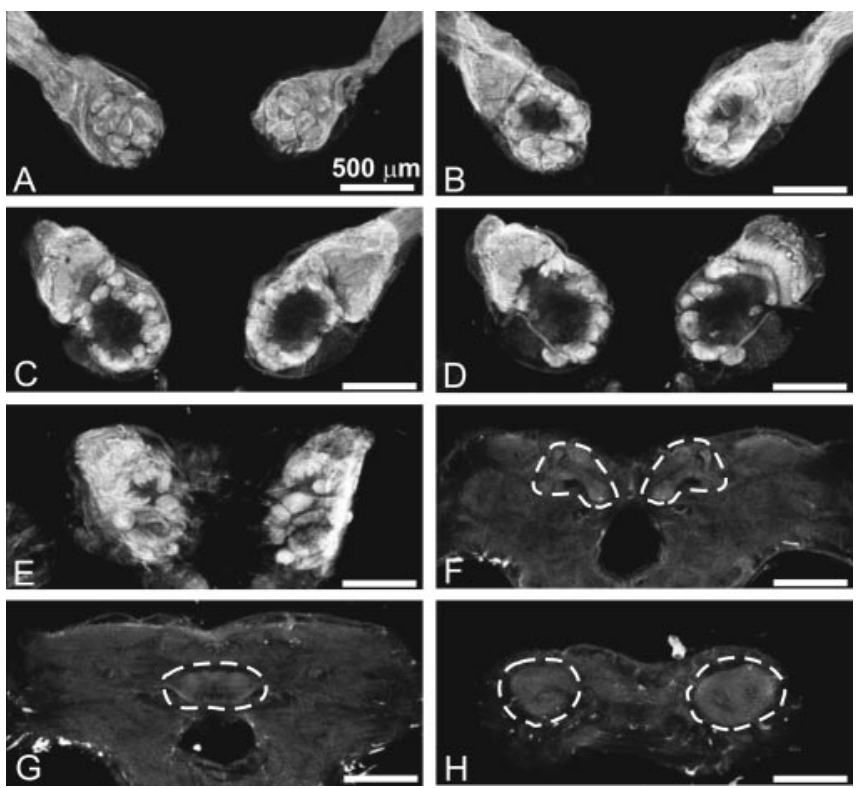

Figure 1. Immunocytochemical labeling of NOS protein in the ALs and central brain. Strong NOS labeling is in most or all of the ORN axons in both ALs, which are shown in serial sections from anterior $(A)$ to posterior $(E)$. The mushroom body lobes ( $F$, dashed outlines), central complex ( $G$, dashed outline), mushroom body calyces ( $H$, dashed outlines), and other parts of the central brain do not express NOS at such high levels.

corrected sequence. To compare the peak signals for the different odorants tested, an average peak signal was calculated for each odorant $(10 \mu \mathrm{g}$ of plant-derived odorants, $1 \mu \mathrm{g}$ of pheromone, and $10 \mu \mathrm{l}$ of control solvent). Also, average peak signals for $10 \mu \mathrm{g}$ of $(+/-)$-linalool before and during treatment with $\mathrm{NO}$-signaling antagonists were calculated. Each response group was compared with its respective control using the Student's $t$ test for independent samples.

To compare the peaks of the signals for the different concentrations of $(+/-)$-linalool and pheromone mixtures tested, an average of five frames at the peak of each signal was calculated from the most active region of interest. Three concentrations of each odorant [10 $\mu \mathrm{g}, 100 \mu \mathrm{g}$,

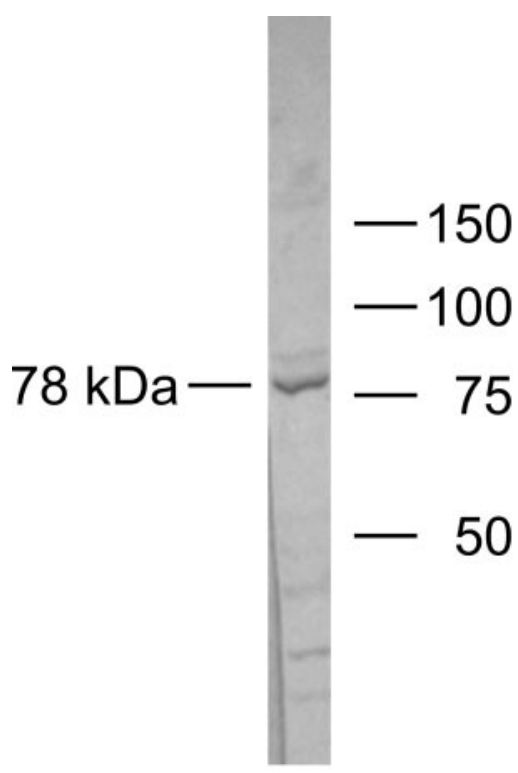

Figure 2. MsGC $\alpha 1$ antiserum recognizes $M s G C \alpha 1$ protein in the adult brain of $M$. sexta by Western blot analysis. The predominant band of $78 \mathrm{kDa}$ recognized by the antiserum matches the predicted size for $\mathrm{MsGC} \alpha 1$ of $78.4 \mathrm{kDa}$. and $1 \mathrm{mg}$ of (+/-)-linalool; $10 \mathrm{ng}, 100 \mathrm{ng}$, and $1 \mu \mathrm{g}$ of pheromone mixture) were compared in one-way ANOVA.

For image presentation, an average of four frames at the peak of the signal (frames 20-23 for plant-derived odorants and frames 23-26 for pheromone) of a bleaching-corrected sequence was calculated, and $F$ was subtracted. The subtracted image was filtered with a spatial average lowpass filter $(15 \times 15$ pixels $)$. Each image was false-color coded to its entire intensity range, except the images of odorant concentration-dependent signals, which were all false-color coded to the intensity range appropriate for the highest concentration to allow unbiased comparison of signals from concentration to concentration.

\section{Results}

\section{Labeling NOS and sGC in AL glomeruli}

To confirm the localization of NOS within the adult olfactory system, we used immunocytochemistry with a universal NOS antiserum. As predicted from in situ hybridization (Nighorn et al., 1998), NOS was found to be expressed strongly in ORNs along the length of the antennal nerve and throughout the glomeruli of the AL (Fig. 1A-E). NOS was present also in other brain structures including the central complex and the lobes and calyces of the mushroom bodies, although the levels of NOS expression were much lower than those found in the ORN axons (Fig. $1 F-$ $H$ ). The high levels of NOS in the primary olfactory system in $M$. sexta suggested that NO signaling plays an important role in the function of the olfactory system. In the AL, NOS-positive ORN axons terminate in all of the sexually isomorphic glomeruli, as well as the male-specific MGC, which is responsible for the initial processing of sensory information about the conspecific female pheromone (Christensen and Hildebrand, 1987). The labeling for NOS was uniformly strong in all of the sexually isomorphic glomeruli but appeared less intense in the MGC (see Fig. 3A). This difference may indicate lower expression of NOS in this glomerular structure, although the MGC neuropil also appears to
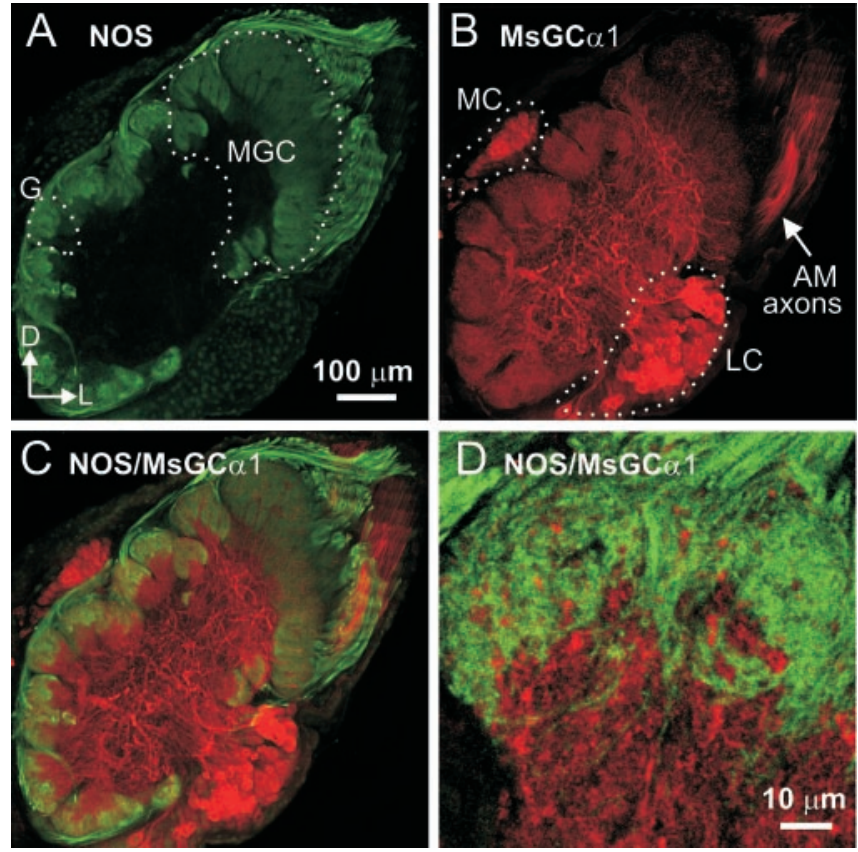

Figure 3. Immunocytochemical labeling of $\mathrm{NOS}$ and $\mathrm{MsGC} \alpha 1$ in the AL. A, All glomeruli (G, dashed outlines) including the MGC, contain NOS-positive ORN axons. $B$, Large subsets of intrinsic AL neurons in the medial and lateral clusters (MC and LC, respectively), and a subset of antennal mechanosensory (AM) axons are MsGC $\alpha 1$ positive. C, The expression patterns for NOS and $M s G C \alpha 1$ are closely apposed in glomeruli. $D, A$ single optical section of an image of a glomerulus shows that NOS and MsGC $\alpha 1$ are not coexpressed in glomeruli. D, Dorsal; L, lateral. 

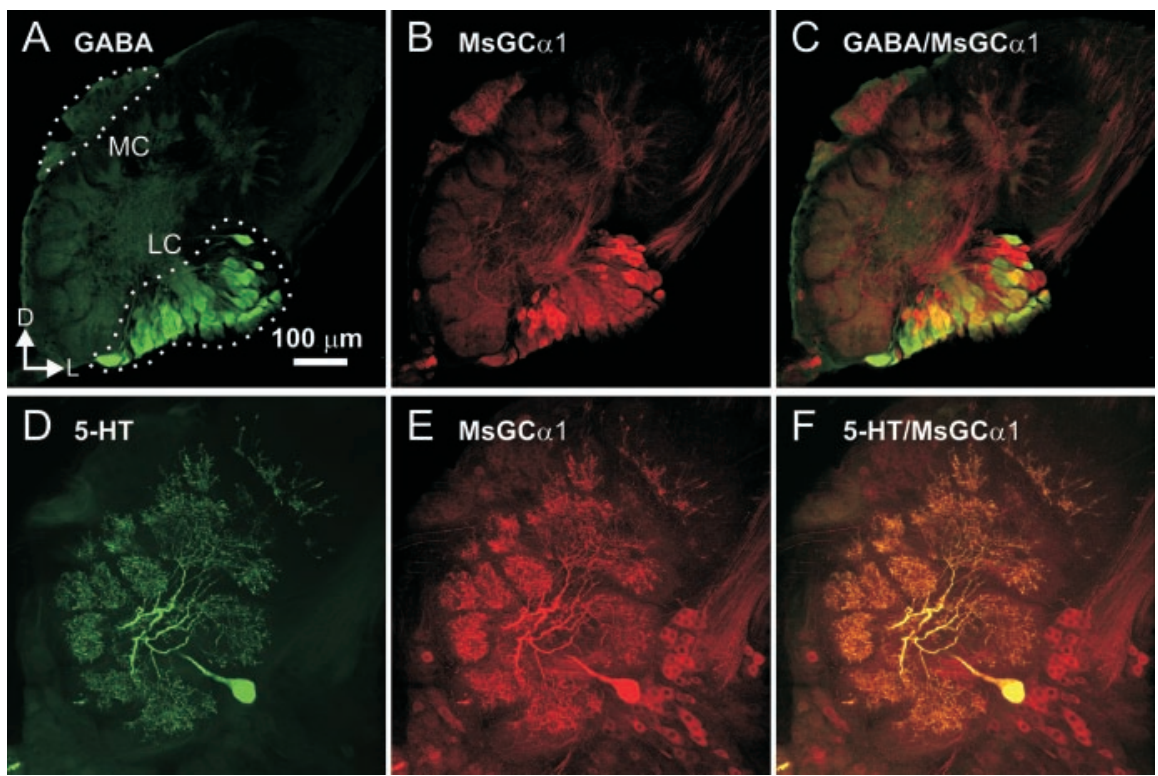

Figure 4. Characterizing $\mathrm{MsGC} \alpha 1$-positive neurons with other immunocytochemical markers. $A$, A large subset of neurons in the lateral cluster (LC), but not the medial cluster (MC), are GABA immunoreactive. $B$, Both clusters contain MsGC $\alpha 1$-positive neurons. C, A small subset of the GABA-immunoreactive neurons also contains MsGC $\alpha 1$ (yellow cell bodies). D, Each AL houses one 5-HT-immunoreactive neuron. E, F, The serotonin-immunoreactive neuron expresses MsGC $\alpha 1$ highly. D, Dorsal; L, lateral.

source of that calcium has not been characterized completely. Together, these data lead to the hypothesis that calcium influx through voltage-gated calcium channels opening in response to action potentials could activate NOS, producing an NO signal in addition to normal synaptic release of neurotransmitter.

\section{Which neurons could be affected by NO?}

Although sGC is not the only target of NO, a better characterization of the subset of neurons that expressed sGC would help to understand the potential functions of odorant-induced NO signals. As seen in Figure 3, sGC is expressed in a subset of $\mathrm{AL}$ neurons that includes most or all of the neurons in the medial cell cluster and a fraction of the neurons in the lateral cell cluster. It is thought that all of the neurons in the medial cell cluster are projection neurons (Kanzaki et al., 1991); thus, projection neurons are likely to be affected by NO. The lateral cell body cluster, in contrast, contains both projection neurons

be less dense than that of the sexually isomorphic glomeruli. All glomeruli, nonetheless, appeared to contain NOS-positive ORN axons. No expression of NOS was seen in the intrinsic AL neurons, indicating that $\mathrm{NO}$ in the AL may be synthesized only by the ORN axons and that NO-mediated communication in glomeruli originates only from the sensory afferents.

To begin identifying the potential targets of $\mathrm{NO}$ within the AL, we generated an anti-peptide antibody against MsGC $\alpha 1$. This antibody specifically labels a protein of the expected size for MsGC $\alpha 1(78 \mathrm{kDa})$ on Western blots (Fig. 2). The immunocytochemical expression pattern of MsGC $\alpha 1$ showed a very interesting contrast to the NOS expression pattern (Fig. 3B). Labeling for MsGC $\alpha 1$ was seen in large subsets of AL neurons in both the medial [housing projection neurons (Kanzaki et al., 1991)] and lateral [housing projection and local interneurons (Hoskins et al., 1986)] cell body clusters. No labeling was seen in the ORN axons, but a subset of axons were labeled in the antennal mechanosensory bundle, which bypasses the AL. Labeling NOS and MsGC $\alpha 1$ together showed the remarkable contrast between the potential NO sources and the potential NO targets (Fig. 3C). Whereas NOS was expressed highly in all glomeruli in the presynaptic ORN axons, MsGC $\alpha 1$ was expressed highly in all glomeruli in postsynaptic neuronal processes originating from large subsets of $\mathrm{AL}$ neurons housed in both the medial and the lateral cell body clusters. The two expression patterns were very closely apposed, yet non-overlapping (Fig. 3D). Together, the patterns suggested strongly that NO-mediated communication could occur between ORNs and intrinsic AL neurons. Although sGC is not likely to be the only target for NO in Manduca, this anatomical arrangement suggests that the odorant responses of sGC-positive neurons could be modulated by NO. The strong expression of NOS in the terminal regions of the ORN axons, coupled with the fact that $M$. sexta NOS activity can be stimulated strongly by calcium (Nighorn et al., 1998), suggested that NO signals could be triggered by action potentials in activated ORNs. In support of this idea, odorant stimulation has been shown to elicit calcium transients within the glomeruli of M. sexta (Hansson et al., 2003), although the and local interneurons. To begin to characterize which subset expressed sGC, we colocalized sGC with serotonin and GABA in double-label experiments. GABA is the neurotransmitter for a large subset of local interneurons and a few projection neurons (Hoskins et al., 1986). We found that MsGC $\alpha 1$ was expressed in only a small subset of GABA-immunoreactive neurons (Fig. $4 A-$ $C)$. Given the small number of neurons that were double-labeled, we were unable to determine whether the small subset of neurons that were both MsGC $\alpha 1$ positive and GABA immunoreactive were local interneurons or projection neurons. Clearly, however, most of the GABAergic local interneurons were not strongly positive for MsGC $\alpha 1$. One clearly identifiable neuron in the lateral cell body packet is the serotonergic neuron. This neuron has its cell body in the lateral cell body packet but projects to all of the glomeruli in the contralateral AL. It also is thought to mediate the signaling of descending information from higher brain centers (Homberg and Hildebrand, 1989). This serotoninimmunoreactive neuron expresses MsGC $\alpha 1$ very strongly (Fig. $4 D-F)$, so it is a likely target for NO. Taken together, the results of our immunocytochemical labeling experiments indicate that $\mathrm{NO}$ signaling could play a role in processing odorant information in glomeruli by mediating intercellular communication between odorant-stimulated ORNs and intrinsic AL neurons. The NOsGC pathway in particular is likely to be important for mediating or modulating communication in a large subset of PNs, a small subset of GABA-immunoreactive neurons, and the single serotonin-immunoreactive neuron.

\section{The dynamics of odorant-stimulated NO signals in AL glomeruli}

To test our hypothesis that odorant-stimulated ORN axons produce NO signals in AL glomeruli, we used the fluorescent NO indicator DAF-FM DA to image the real-time odorant responses of M. sexta ALs. In cells, DAF-FM DA is hydrolyzed to DAF-FM, which reacts irreversibly with $\mathrm{NO}$ (or products of NO metabolism) in a concentration-dependent manner to become a highly fluorescent benzotriazole derivative (Kojima et al., 1999). In an 
experimental setup analogous to that used previously for imaging calcium responses in M. sexta (Hansson et al., 2003), the dye was loaded into the Als, and the responses of the visible surface glomeruli were recorded in an individual AL while we stimulated the ipsilateral antenna with odorants. NO signals were imaged for four biologically relevant plant odorants [geraniol, (+/-)linalool, 1-octanol, and PAA], each dissolved in paraffin oil, and synthetic mimics of the two, main components $[E 10, Z 12$ hexadecadienal (bombykal), and EEZ] of the conspecific female pheromone, dissolved separately or as a 1:1 mixture in cyclohexane.

The first response examined was to (+/-)-linalool. Stimulation with this odorant resulted in a strong and reproducible pattern of fluorescence in the AL. There was a peak focus of fluorescence, about the size expected for a glomerulus, that was slightly lateral and ventral to the center of the AL (Fig. $5 A$ ). In addition to this strong peak of fluorescence, there were two other areas that were close by but more ventral and showed a less intense fluorescence. These patterns were highly reproducible with multiple stimulations in the same AL and in all animals tested with this odorant $(n=6)$. Paraffin oil-only control stimuli did not evoke NO signals. In Figure $5 B$, the dynamics of the NO signal shown in Figure $5 A$ are compared with the dynamics of the relative changes in fluorescence $(\mathrm{d} F / F)$ for a single control stimulus given in the same experiment. The peak fluorescence occurred $\sim 2.6 \mathrm{sec}$ after the onset of odorant stimulation. To test the specificity of DAF-FM DA, we bathed the ALs in saline that contained both an NO scavenger, C-PTIO, and an NOS inhibitor, L-NAME. We compared the average peak $\mathrm{d} F / F$ in the most active glomerulus responding to $(+/-)$-linalool before and during treatment (Fig. $5, G$ and $H$, respectively). The average peak $\mathrm{d} F / F$ was reduced significantly by these NO-signaling antagonists (see the legend for Fig. 5). Note that these images are from a different animal and that the field of view is at an angle slightly different from that in Figure $5 A$.

The evoked patterns of NO signals varied for each odorant tested. Stimulation of the antenna with geraniol resulted in a small focus of strongly increased fluorescence just dorsal to the center of the AL and relatively widespread areas of weakly increased fluorescence that were lateral to the main focus (Fig. 5C). Stimulation with 1-octanol, in contrast, resulted in a very similar area of strongly increased fluorescence, but the weakly fluorescent areas were more focused, and all were more medial than for geraniol stimulation (Fig. 5D). Stimulation with PAA resulted in peak fluorescence in two closely related areas in the ventromedial part of the AL and a pattern of weak fluorescence that was lateral to the main foci. Finally, after moving the AL so that the MGC area was in the field of view of the microscope, we found that pheromone stimulation evoked a large area of activity in what is presumably the MGC (Fig. $5 F$ ). This result is consistent with both electrophysiological and calcium imaging studies of pheromone-stimulated activity in the MGC of $M$. sexta males (Christensen and Hildebrand, 1987; Hansson et al., 2003). The four non-pheromonal odorants did not evoke significant increases in fluorescence in the visible portion of the MGC area (Fig. 5A, C-E, white dotted outlines), where a component of the pheromone induced a strong fluorescent signal (Fig. $5 F$ ). Likewise, pheromone stimulation did not evoke significant increases in fluorescence outside of the MGC area, although the field of view (Fig. $5 F$ ) used to image pheromone responses did not include many of the sexually isomorphic glomeruli at the surface of the lobe.

For quantitative analysis of the responses, we examined the magnitude and timing of the peak signal for each odorant. To
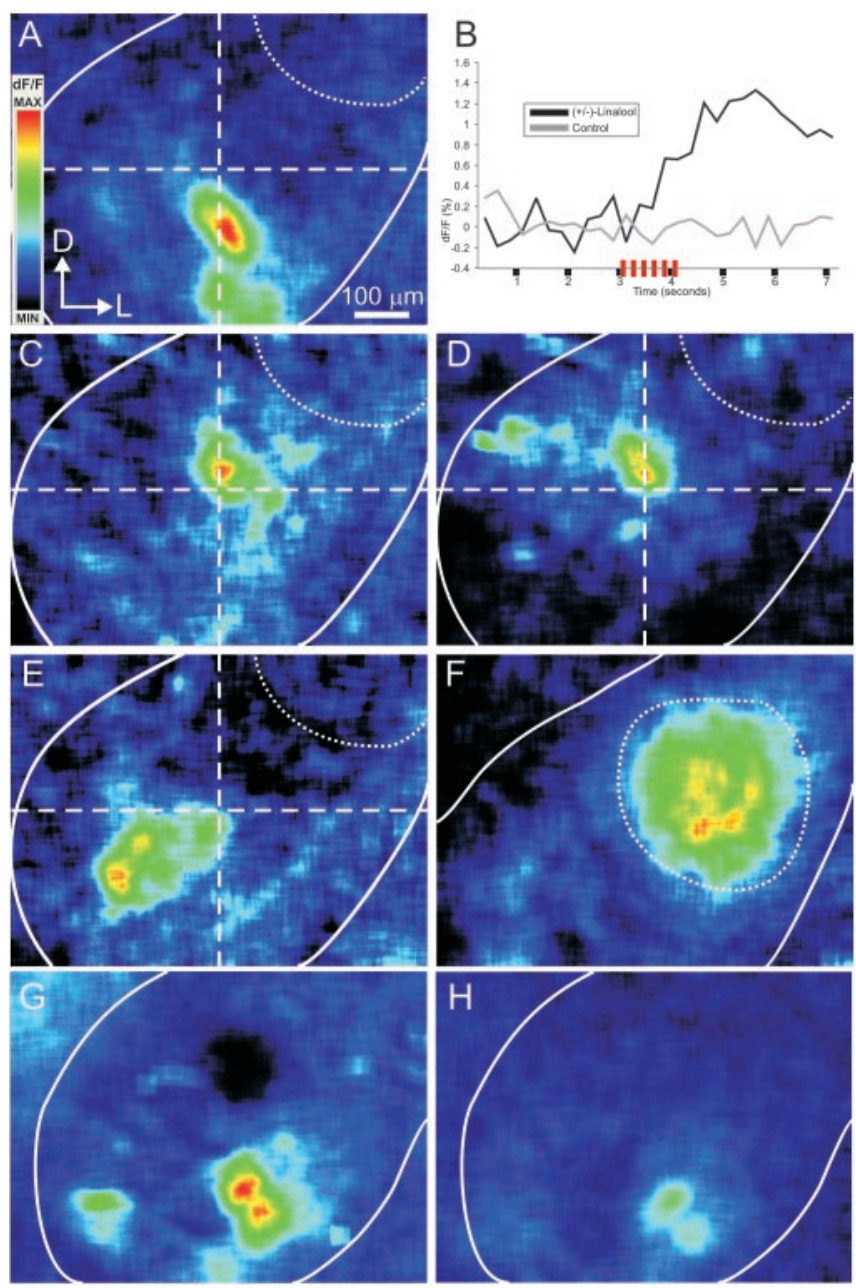

Figure 5. Typical NO signals for the odorants tested. The images show the relative change $(\mathrm{d} F / F)$ in activity after stimulation. Each image is an average of four frames at the peak of the signal. Each image is false-color coded and scaled to its entire intensity range. The solid white outlines are an estimate of the AL border. The dashed white lines mark the center of the image. The dotted white outlines are an estimate of the visible MGC area. $A$, NO signals in a single male AL for $10 \mu \mathrm{g}$ of (+/-)-linalool. $B, A$ graph of the temporal dynamics of the peak of the NO signal for $A$ is shown relative to control. The stimulus pulses are indicated by red bars. N0 signals in the same AL for geraniol (C), 1-octanol (D), PAA ( $E)$, and $1 \mu \mathrm{g}$ of EEZ $(F) . G, H$, In a different male AL, the NO signal for $10 \mu \mathrm{g}$ of $(+/-)$-linalool is reduced by C-PTIO and L-NAME. These pharmacological antagonists of NO signaling significantly reduced the average peak $\mathrm{d} F / F$ in three animals tested $(1.11 \pm 0.21 \%$ before treatment, $n=6$ stimulations; $0.88 \pm 0.23 \%$ during treatment, $n=6$ stimulations; $p<0.001$ ). Note that this level of reduction $(\sim 21 \%)$ is similar to that reported in the literature for these antagonists of NO signaling (Pittner et al., 2003). D, Dorsal; L, lateral.

measure the peak response, the average peak $\mathrm{d} F / F$ from a $1 \mathrm{sec}$ time period after stimulus onset for each individual response was compared with controls. All odorants tested, pheromone included, evoked a statistically significant average $\mathrm{d} F / F$ of $\sim 1 \%$ relative to solvent-only controls (Fig. $6 \mathrm{~A}$ ). We next examined the temporal dynamics of the peak responses by grouping them according to the identity of the odorant stimulus and calculating an average NO signal for each odorant. We found that all odorants tested evoked the onset of a signal almost immediately after the onset of the stimulus (Fig. $6 B, C$ ). We found also that $10 \mu \mathrm{g}$ of a plant odorant stimulus, in general, evoked an absolute peak $\mathrm{d} F / F$ that occurred $2.6 \mathrm{sec}$ after the onset of the stimulus (Fig. $6 \mathrm{~B}$ ). The timing of NO signals in the sexually isomorphic glomeruli appeared to be independent of the identity of the stimulus. In con- 


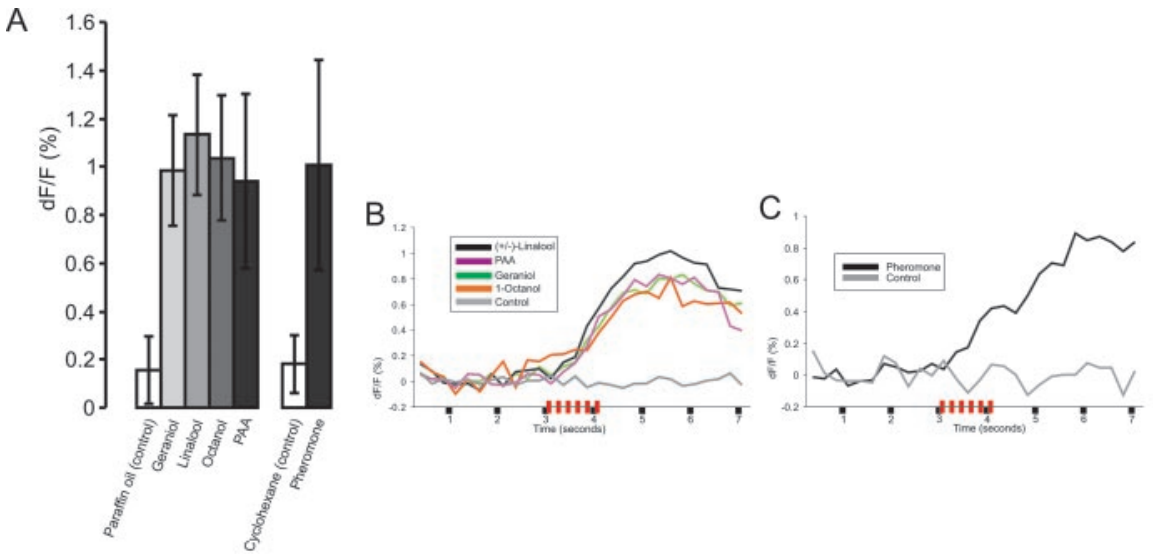

Figure 6. Peak magnitudes and temporal dynamics of $\mathrm{d} F / F$. A, Average peak magnitude of odorant-evoked N0 signals, which were calculated from the most activated glomerulus of each response. $B$, Temporal dynamics of the averaged signals for each plant odorant (10 $\mu \mathrm{g}$ ) tested. C, Temporal dynamics of the averaged signals for $1 \mu \mathrm{g}$ of pheromone. Red bars indicate stimulus pulses. Paraffin oil, $n=20$ stimulations in seven animals; geraniol, $n=11$ responses in seven animals; (+/-)-linalool, $n=14$ responses in six animals; 1 -octanol, $n=8$ responses in five animals; PAA, $n=13$ responses in seven animals; cyclohexane, $n=$ 8 stimulations in three animals; pheromone, $n=8$ responses in three animals. All odorant-evoked signals were significantly different from controls ( $p<0.0001$ for all).

trast, the absolute peak $\mathrm{d} F / F$ induced by $1 \mu \mathrm{g}$ of pheromone occurred $\sim 2.85 \mathrm{sec}$ or more after stimulus onset (Fig. $6 C$ ). The reason for this delay is not clear but may be attributable to the lower concentration of stimulus used to evoke a response, or may reflect a lower density of the neuropil in the MGC.

Odorant-stimulated NO signals are concentration dependent We next examined the effects of odorant concentration on the NO signals in the AL. The responses to decadic concentration series of $(+/-)$-linalool and of the pheromone mixture were examined. For linalool, we tested a concentration series from 10 ng to $1 \mathrm{mg}$, but significant responses were consistently obtained only at concentrations of $10 \mu \mathrm{g}$ and above (Fig. $7 A-D$ ). This finding agreed with calcium imaging experiments that showed that, in general, $10 \mu \mathrm{g}$ of a plant odorant stimulus was the threshold for consistent, reproducible signals in M. sexta (Hansson et al., 2003). Increasing concentrations of the odorant evoked increasingly intense NO signals in the most active glomerulus as well as in less active glomeruli. Additionally, higher concentrations recruited activity in areas of the AL adjacent to or proximal to the most active foci. To quantify the effect of odorant concentration on the intensity of the NO signal, we calculated an average peak $\mathrm{d} F / F$ of the most active area for each response and grouped the responses according to concentration. The responses to each concentration were then compared with the responses to the other concentrations in a one-way ANOVA (Fig. 7D). The differences in average peak $\mathrm{d} F / F$ across the concentration groups were attributable to the changes in odorant concentration. Thus, quantification of the highest-intensity pixels showed clearly increasing signal intensity in response to increasing odorant concentration.

Next, we used the pheromone mixture to test whether pheromone-evoked NO signals in the MGC area depended on stimulus concentration. The pheromone mixture was tested in a decadic concentration series from $1 \mathrm{ng}$ to $1 \mu \mathrm{g}$, although the males did not respond detectably to the lowest concentration (1 ng). Increasing concentrations of pheromone evoked increasingly greater NO signals in the MGC area, but the overall area of the response did not appear to increase (Fig. $7 E-H$ ). This suggests that high concentrations of pheromone did not appear to recruit activity in areas of the $\mathrm{AL}$ other than the MGC area. Because we had focused on the MGC area, however, we could see only a small portion of the rest of the visible AL and could have missed other responses. Quantification of the intensity of the most active areas of the MGC showed a significant increase in the intensity of the signal attributable to the increased odor concentration (Fig. $7 H$ ).

\section{Discussion}

This study examined the role of NO signaling in the adult olfactory system of $M$. sexta. We showed that the highest levels of NOS expression in the M. sexta brain were in the ORNs and that each glomerulus contained NOS-positive ORN axons. Moreover, we found that sGC was present in a subset of AL neurons that included projection neurons, a serotonin-immunoreactive neuron, and a subset of GABA-immunoreactive neurons. More importantly, we used the marker DAF-FM DA to show that odorant stimulation evokes NO production in the AL. The NO signals were focal and reproducible. These focal NO signal patterns varied for each odorant and were decreased by pharmacological antagonists of NO signaling. Finally, we showed that the patterns of NO production were concentration dependent. Increasing concentrations of odorants stimulated increasing intensities of NO signals. Increasing concentrations of non-pheromonal odorants evoked an increase in the area of the AL that responded to stimulation.

\section{DAF-FM DA as a marker for NO}

The development of diaminofluoresceins as markers for $\mathrm{NO}$ (Kojima et al., 1998) was met originally with great enthusiasm, but subsequent studies showed that they might react more sensitively with nitrates or other products of NO metabolism rather than NO itself (Roychowdhury et al., 2002) and may interact with ascorbic acid (Zhang et al., 2002). In our case, these potential complications do not change the analysis of the results significantly. It is very unlikely that ascorbic acid levels would change acutely and focally in response to odorant stimulation. The question of whether or not the marker reacts to NO or NO metabolites also does not detract from our conclusions. Whether the fluorescence that we saw was attributable to primary or secondary reactions to $\mathrm{NO}$, the presence of $\mathrm{NO}$ in response to odorant stimulation is still strongly evident. The localization patterns of NOS in the ORN axons and the decrease in odorant-evoked fluorescence in the presence of NO-signaling inhibitors suggests strongly the presence of $\mathrm{NO}$ in the $\mathrm{AL}$ as a response to odorant stimulation.

\section{The patterns of odorant-evoked NO signals}

Odorant mapping studies using fluorescent calcium indicators, voltage-sensitive dyes, or 2-deoxyglucose (Linster et al., 2001; Meister and Bonhoeffer, 2001; Wachowiak and Cohen, 2001) have shown in many different organisms that odorants evoke focal patterns of glomerular activity. In moths, including $M$. sexta, odorants elicit focal patterns of calcium transients in $\mathrm{AL}$ glomeruli (Galizia et al., 2000; Carlsson et al., 2002; Hansson et al., 2003). Our real-time recordings using the fluorescent NO dye 
DAF-FM DA show, for the first time in any organism, that odorants can also evoke focal NO signals at the first synaptic neuropil in the olfactory system. The fact that the signals were focal and spatially distinct for different odorants suggested that they were, in fact, glomerulus specific. This study, however, did not provide sufficient resolution of the glomerular borders to show explicitly that the strong foci of increased fluorescence corresponded exactly to olfactory glomeruli. In addition to the strongly fluorescent areas, each odorant also evoked areas of weaker fluorescence that were generally close to the main foci. These could be either the result of diffusion of the NO signal (Philippides et al., 2000 ) or production of NO within adjacent, less active glomeruli. Presumably, the ORNs projecting to these adjacent glomeruli were stimulated only weakly by the odorant tested. Although diffusion of the NO signal cannot be ruled out as a cause, the patterns of odorant-induced fluorescence are strongly suggestive of the idea that the effect is attributable to activation of ORNs. This is particularly evident when the patterns of geraniol and 1-octanol are compared. Although the strong focus of fluorescence is very similar for both, their patterns of weaker fluorescence are completely different. It is not likely that simple diffusion could account for this phenomenon. It has also been suggested that the glial cells surrounding each glomerulus provide a significant barrier to the diffusion of small molecules (Goriely et al., 2002), but the efficacy of glial cells as an NO sink has not been tested. Finally, the patterns of fluorescence seen in Figure 7 show no evidence of a time-dependent spread that would indicate diffusion. Instead, the areas of weaker fluorescence are seen at very early time points but simply fail to reach the peak intensity of the main foci.

The NO signals that we recorded were found to be concentration dependent. This is consistent with the results from calcium imaging studies. In moths, calcium signals were also found to depend on the concentration of the odorant stimulus (Galizia et al., 2000; Carlsson and Hansson, 2003). In our NO imaging studies, increased concentrations of linalool evoked increases in the peak levels of fluorescence and increases in the areas of the AL that were both strongly and weakly fluorescent. Increases in concentration of the pheromone mixture, in contrast, resulted in increases in signal intensity without an apparent increase in signal area. These observations are consistent with the idea that higher concentrations of non-pheromonal odorants activated more types of odorant receptors and, therefore, more glomeruli. Increases in the concentration of the pheromone mixture, in contrast, being orders of magnitude lower in concentration than the concentrations tested for linalool, evoked stronger activation of the pheromone-sensitive ORNs only. If diffusion outside of glomeruli occurred, we would expect that regions of the AL near the MGC would become fluorescent as $\mathrm{NO}$ diffused out of the MGC. All of these data together indicate that active ORNs produce NO focally within AL glomeruli.

\section{The function of NO in the AL}

What is the function of NO in AL glomeruli? This question, in part, is dependent highly on the timing of NO signals. We see the first detectable increase in fluorescence between 250 and 500 msec after stimulation. Given our experimental setup, it is impossible to tell when exactly NO is produced relative to synaptic transmission. If we assume that the same calcium influx that accompanies the synaptic release of neurotransmitter also activates NOS, it is likely that NO synthesis does not occur as quickly as the fusion of synaptic vesicles. This would indicate that NO signals, although accompanying normal synaptic transmission, 
would be slower and would not affect the initial postsynaptic response to the odorant. Rather, they would affect the continued or subsequent responses to the odorant. This, however, needs to be tested explicitly.

The effects of NO signals are likely to be determined by which neurons are affected and by which molecular mechanisms mediate the signals. The best characterized target of NO is sGC, but there are many other potential targets, including olfactory cyclic nucleotide gated channels (Broillet and Firestein, 1996) and GABA receptors (Fukami et al., 1998), both of which are likely to be expressed in the AL. Different molecular targets of NO might mediate different effects on AL neuron activity. The identity of the affected neuron is also important. Because projection neurons express high levels of sGC, their activity may be sensitive to the levels of NO. In this case, only the projection neurons that come from the active glomerulus would be affected. Also, if the excitability of the serotonergic neuron is affected by NO-sGC signaling, it could affect levels of serotonin and, therefore, global levels of activity in the contralateral AL. In any case, the high expression of NOS in ORN axons in every glomerulus and the focal production of $\mathrm{NO}$ within $\mathrm{AL}$ glomeruli suggest that $\mathrm{NO}$ signaling plays an important role in the processing of odorant information in the AL of M. sexta.

\section{References}

Alonso JR, Porteros A, Crespo C, Arevalo R, Brinon JG, Weruaga E, Aijon J (1998) Chemical anatomy of the macaque monkey olfactory bulb: NADPH-diaphorase/nitric oxide synthase activity. J Comp Neurol 402:419-434.

Bredt DS, Glatt CE, Hwang PM, Fotuhi M, Dawson TM, Snyder SH (1991) Nitric oxide synthase protein and mRNA are discretely localized in neuronal populations of the mammalian CNS together with NADPH diaphorase. Neuron 7:615-624.

Breer H, Shepherd GM (1993) Implications of the NO/cGMP system for olfaction. Trends Neurosci 16:5-9.

Broillet MC, Firestein S (1996) Direct activation of the olfactory cyclic nucleotide-gated channel through modification of sulfhydryl groups by NO compounds. Neuron 16:377-385.

Carlsson MA, Hansson BS (2003) Dose-response characteristics of glomerular activity in the moth antennal lobe. Chem Senses 28:269-278.

Carlsson MA, Galizia CG, Hansson BS (2002) Spatial representation of odours in the antennal lobe of the moth Spodoptera littoralis (Lepidoptera: Noctuidae). Chem Senses 27:231-244.

Christensen TA, Hildebrand JG (1987) Male-specific, sex pheromoneselective projection neurons in the antennal lobes of the moth Manduca sexta. J Comp Physiol [A] 160:553-569.

Elphick M, Rayne R, Riveros-Moreno VV, Moncada S, Shea M (1995) Nitric oxide synthesis in locust olfactory interneurones. J Exp Biol 198:821-829.

Fukami S, Uchida I, Mashimo T, Takenoshita M, Yoshiya I (1998) Gamma subunit dependent modulation by nitric oxide (NO) in recombinant GABAA receptor. NeuroReport 9:1089-1092.

Galizia CG, Sachse S, Mustaparta H (2000) Calcium responses to pheromones and plant odours in the antennal lobe of the male and female moth Heliothis virescens. J Comp Physiol [A] 186:1049-1063.

Gelperin A, Flores J, Raccuia-Behling F, Cooke IR (2000) Nitric oxide and carbon monoxide modulate oscillations of olfactory interneurons in a terrestrial mollusk. J Neurophysiol 83:116-127.

Gibson NJ, Nighorn A (2000) Expression of nitric oxide synthase and solu- ble guanylyl cyclase in the developing olfactory system of Manduca sexta. J Comp Neurol 422:191-205.

Goriely AR, Secomb TW, Tolbert LP (2002) Effect of the glial envelope on extracellular $\mathrm{K}^{+}$diffusion in olfactory glomeruli. J Neurophysiol 87:1712-1722.

Hansson BS, Carlsson MA, Kalinova B (2003) Olfactory activation patterns in the antennal lobe of the sphinx moth, Manduca sexta. J Comp Physiol [A] 189:301-308.

Homberg U, Hildebrand JG (1989) Serotonin-immunoreactive neurons in the median protocerebrum and suboesophageal ganglion of the sphinx moth Manduca sexta. Cell Tissue Res 258:1-24.

Hopkins DA, Steinbusch HW, Markerink-van Ittersum M, De Vente J (1996) Nitric oxide synthase, cGMP, and NO-mediated cGMP production in the olfactory bulb of the rat. J Comp Neurol 375:641-658.

Hoskins SG, Homberg U, Kingan TG, Christensen TA, Hildebrand JG (1986) Immunocytochemistry of GABA in the antennal lobes of the sphinx moth Manduca sexta. Cell Tissue Res 244:243-252.

Kanzaki R, Arbas EA, Hildebrand JG (1991) Physiology and morphology of protocerebral olfactory neurons in the male moth Manduca sexta. J Comp Physiol [A] 168:281-298.

Kendrick KM, Guevara-Guzman R, Zorrilla J, Hinton MR, Broad KD, Mimmack M, Ohkura S (1997) Formation of olfactory memories mediated by nitric oxide. Nature 388:670-674.

Kojima H, Nakatsubo N, Kikuchi K, Kawahara S, Kirino Y, Nagoshi H, Hirata Y, Nagano T (1998) Detection and imaging of nitric oxide with novel fluorescent indicators: diaminofluoresceins. Anal Chem 70:2446-2453.

Kojima H, Urano Y, Kikuchi K, Higuchi T, Hirata Y, Nagano T (1999) Fluorescent indicators for imaging nitric oxide production. Angew Chem Int Ed Engl 38:3209-3212.

Linster C, Johnson BA, Yue E, Morse A, Xu Z, Hingco EE, Choi Y, Choi M, Messiha A, Leon M (2001) Perceptual correlates of neural representations evoked by odorant enantiomers. J Neurosci 21:9837-9843.

Meister M, Bonhoeffer T (2001) Tuning and topography in an odor map on the rat olfactory bulb. J Neurosci 21:1351-1360.

Muller U, Hildebrandt H (1995) The nitric oxide/cGMP system in the antennal lobe of Apis mellifera is implicated in integrative processing of chemosensory stimuli. Eur J Neurosci 7:2240-2248.

Murakami M, Watanabe S, Inoue T, Kirino Y (2004) Odor-evoked responses in the olfactory center neurons in the terrestrial slug. J Neurobiol 58:369-378.

Nighorn A, Gibson NJ, Rivers DM, Hildebrand JG, Morton DB (1998) The nitric oxide-cGMP pathway may mediate communication between sensory afferents and projection neurons in the antennal lobe of Manduca sexta. J Neurosci 18:7244-7255.

Philippides A, Husbands P, O’Shea M (2000) Four-dimensional neuronal signaling by nitric oxide: a computational analysis. J Neurosci 20:1199-1207.

Pittner J, Liu R, Brown R, Wolgast M, Persson AEG (2003) Visualization of nitric oxide production and intracellular calcium in juxtamedullary afferent arteriolar endothelial cells. Acta Physiol Scand 179:309-317.

Roychowdhury S, Luthe A, Keilhoff G, Wolf G, Horn TF (2002) Oxidative stress in glial cultures: detection by DAF-2 fluorescence used as a tool to measure peroxynitrite rather than nitric oxide. Glia 38:103-114.

Sanes JR, Hildebrand JG (1976) Origin and morphogenesis of sensory neurons in an insect antenna. Dev Biol 51:300-319.

Wachowiak M, Cohen LB (2001) Representation of odorants by receptor neuron input to the mouse olfactory bulb. Neuron 32:723-735.

Zhang X, Kim WS, Hatcher N, Potgieter K, Moroz LL, Gillette R, Sweedler JV (2002) Interfering with nitric oxide measurements. 4,5-diaminofluorescein reacts with dehydroascorbic acid and ascorbic acid. J Biol Chem 277:48472-48478. 